\title{
Hybrid Light-Emitting Diodes from Microcontact-Printing Double-Transfer of Colloidal Semiconductor CdSe/ZnS Quantum Dots onto Organic Layers**
}

\author{
By Aurora Rizzo, Marco Mazzeo, Marco Palumbo, Gianluca Lerario, Stefania D’Amone, \\ Roberto Cingolani, and Giuseppe Gigli*
}

Colloidal semiconductor nanocrystals have been attracting increasing technological interest for applications in lighting and flat-panel display applications. ${ }^{[1-7]}$ In particular, continuing improvements in synthetic methods have enabled the design of high-quality quantum dot (QD) structures with photoluminescence (PL) ranging across the visible spectrum, a PL full width at half maximum (FWHM) less than $30 \mathrm{~nm}$, and a PL efficiency higher than $50 \% .^{[6-10]}$

Several approaches including wet deposition techniques such as spin-coating, ${ }^{[1-6]}$ drop-casting, ${ }^{[11]}$ electrodeposition, ${ }^{[12]}$ and Langmuir-Schafer methods ${ }^{[13,14]}$ have been used to fabricate QD thin films. Device structures incorporating QDs have also been fabricated using some of these approaches. Hybrid organic-inorganic light-emitting diodes (LEDs) have been fabricated using host-guest systems comprising a polymer doped with nanocrystals. ${ }^{[2]}$ In another approach, trilayer hybrid polymer-QD LEDs have been fabricated by sandwiching several monolayers of $\mathrm{CdSe} / \mathrm{ZnS}$ nanocrystals between a hole-conducting polymer and an electron-conducting oxadiazole derivative. ${ }^{[4]}$ In this case, all the layers have been deposited by spin-coating from very dissimilar solvents, for example, CdSe/ZnS QDs have been deposited from aqueous solution, whereas polymers have been deposited from organic solvents. High-efficiency electroluminescent devices have been reported by Coe-Sullivan et al. using the phase-separation technique. ${ }^{[1,5]}$ These authors have achieved the phase separation of small organic molecules and aliphatically capped QDs during the spin-coating of blends of such materials. However, a major drawback of all these device structures is that they are limited by the chemical properties of the materials and the

[*] Dr. G. Gigli, A. Rizzo,* Dr. M. Mazzeo, Dr. M. Palumbo, ${ }^{[+]}$Dr. G. Lerario, Dr. S. D'Amone, Dr. R. Cingolani

NNL, National Nanotechnology Laboratory of CNR-INFM Distretto Tecnologico ISUFI

Dipartimento di Ingegneria dell'Innovazione

Università del Salento

Via Arnesano Km 5, 1-73100 Lecce (Italy)

E-mail: gigli@in.dii.unile.it

[+] Present address: Advanced Technology Institute, Nano-Electronics Centre, University of Surrey, Guildford GU2 7XH, UK

[**] The authors gratefully acknowledge Sonia Carallo for etching indium tin oxide (ITO) substrates, Gianmichele Epifani for performing oxygen plasma treatments of ITO substrates, Luigi Carbone for assistance with transmission electron microscopy imaging, and Barbara Cortese and Claudia Piliego for help with scanning electron microscopy imaging. deposition techniques employed to fabricate these films. In practical terms, this sets stringent restrictions on the organic materials that can be used to fabricate the hole-transporting and hole-injection layers (HTL and HIL, respectively), which are critical for the optimization of the device emission. ${ }^{[1,4,6]}$

Unlike organic small molecules, it is not possible to deposit QDs by thermal evaporation owing to their high molecular weight. Consequently, the separation of the QD active layer from organic transport layers and the fabrication of multilayered organic-inorganic structures still remains a significant challenge. The absence of an appropriate QD deposition technique has strongly limited the implementation of hybrid LEDs in technologically relevant heterojunction devices. This technology is expected to yield significant improvements in the efficiency and lifetime of the devices.

Microcontact printing $(\mu \mathrm{CP})$ offers a promising alternative to the above-described QD wet deposition methods. This approach has already been used for the deposition of uniform closepacked monolayer and bilayer arrays of alkanethiol-capped $\mathrm{Au}$ nanoparticles. ${ }^{[14]}$ In another example, $\mu \mathrm{CP}$ has been used for the deposition of $\left(\mathrm{Cd}_{x} \mathrm{Zn}_{1-x} \mathrm{Se}\right) \mathrm{Cd}_{y} \mathrm{Zn}_{1-y} \mathrm{~S}$ core/shell nanocrystals on $4,4^{\prime}-N, N^{\prime}$-diphenylcarbazole (CBP) for the fabrication of green LEDs. ${ }^{\left[{ }^{[7}\right.}$ Moreover, a combination of $\mu \mathrm{CP}$ and covalent linkage has been used for the fabrication of a selectively patterned monolayer assembly of Co nanoparticles. ${ }^{[15]}$

In the standard $\mu \mathrm{CP}$ technique, a poly(dimethylsiloxane) (PDMS) stamp is inked with the relevant material, which is then transferred onto a solid substrate by achieving conformal contact of the stamp pad and the substrate. ${ }^{[16]}$ PDMS is an elastomer that is able to make conformal contact with surfaces over relatively large areas, thus enabling the patterning of micrometer-sized features. One of the most important drawbacks of the PDMS approach, which is especially relevant to the deposition of colloidal semiconductor nanocrystals, is that PDMS is readily swelled by non-polar solvents such as toluene and chloroform that are commonly used to disperse nanocrystals. ${ }^{[16]}$ Consequently, conformal contact between the stamp and the substrate becomes difficult if not impossible. In one approach to overcoming this problem, Coe-Sullivan et al. have modified the surface of the PDMS stamp by coating it with a chemical-vapor-deposited Parylene-C layer. ${ }^{[17]}$ This layer prevents the stamp from swelling, but the need for this deposition step essentially complicates and adds significant cost to the $\mu \mathrm{CP}$ process. Since facile fabrication and rapid processing are two of the most significant advantages of the 
$\mu \mathrm{CP}$ method, it would be ideal to develop an alternative approach that does involve any complex fabrication steps.

In this context, we have developed a dry, simple, and inexpensive deposition technique to transfer colloidal semiconductor QDs onto organic substrates. We refer to this process as $\mu \mathrm{CP}$ double-transfer of colloidal semiconductor nanocrystals. This procedure combines the ease of wet processing techniques with the possibility of growing a heterojunction QD-based LED via thermal evaporation. Figure 1 shows a schematic depiction of the double-transfer process. In the first step, colloidal QDs have been deposited from toluene solution onto a cleaned float glass substrate. Subsequently, after evaporation of the solvent, a PDMS stamp pad has been brought into conformal contact with the surface of the nanocrystal film. The PDMS stamp has then been peeled away, and the nanocrystals have been transferred to the stamp surface. Finally, the QDs have been transferred via a singlestep $\mu \mathrm{CP}$ process from the PDMS stamp to the surface of a previously evaporated organic thin film.

Drop-casting represents the simplest approach for the deposition of a QD solution onto a desired substrate. The degree of order in the resulting assembly is thought to be mainly determined by the rate of solvent evaporation. In this paper, we show that slowing down the evaporation of the solvent, in our case toluene, and preventing it from rapidly dewetting the surface is critical for achieving successful double transfer. Slow evaporation allows nanocrystals to diffuse and self assemble

1

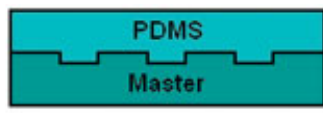

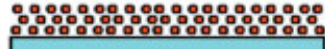
Glass
2

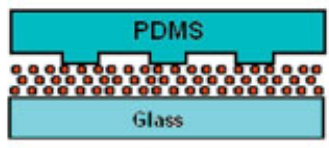

3

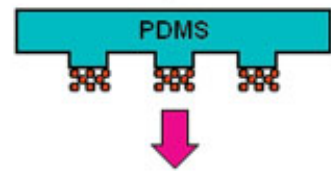

4

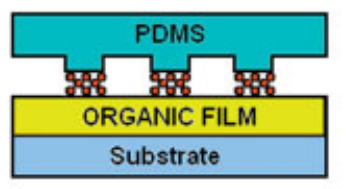

5

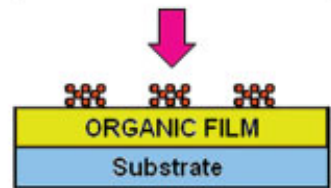

Figure 1. Schematic illustration of the procedure used to transfer colloidal nanocrystals onto thin organic films. into large-area periodic structures (exhibiting long-range order) with relatively few defects. ${ }^{[11]}$

In order to slow down the evaporation process after deposition of the nanocrystals, the substrates have been placed in an over-saturated environment of toluene vapor. The quality of the drop-cast films has been verified by transmission electron microscopy (TEM) imaging. Figure 2 compares two samples obtained by the deposition of $20 \mu \mathrm{L}$ of a QD solution onto a TEM grid in an over-saturated toluene environment (Fig. 2a) and under ambient conditions (Fig. 2b). After slow dewetting of the solvent (about $30 \mathrm{~min}$ ), the nanocrystals form a compact layer extending over several micrometers (Fig. 2a). In contrast, upon rapid solvent evaporation $(<5 \mathrm{~min})$ the film is characterized by the formation of multilayer domains and microscopic voids (Fig. 2b). The deposition of a uniform QD layer on the surface of the organic film is of paramount importance for the proper functioning of hybrid LED devices. ${ }^{[1,5]}$

The sample preparation process developed here involves several steps. First, the QD films have been deposited onto cleaned float glass substrates and dried in an oversaturated environment. Subsequently, flat or patterned PDMS stamps

a)

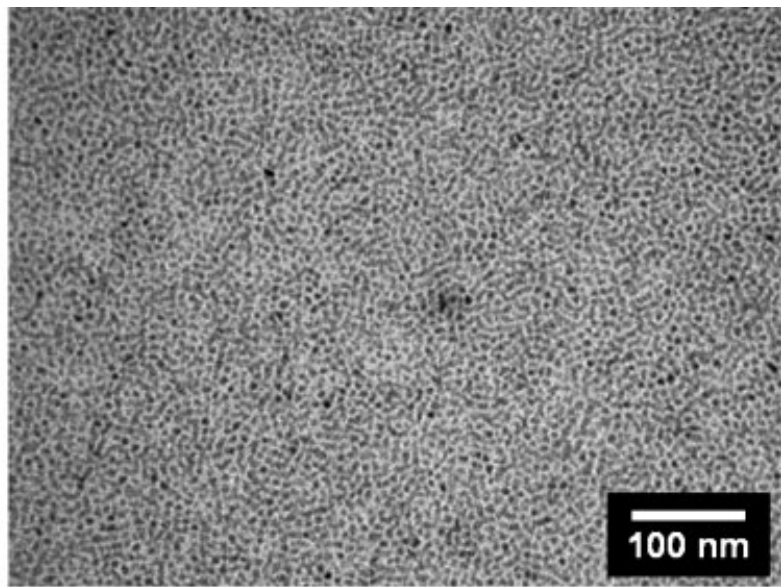

b)

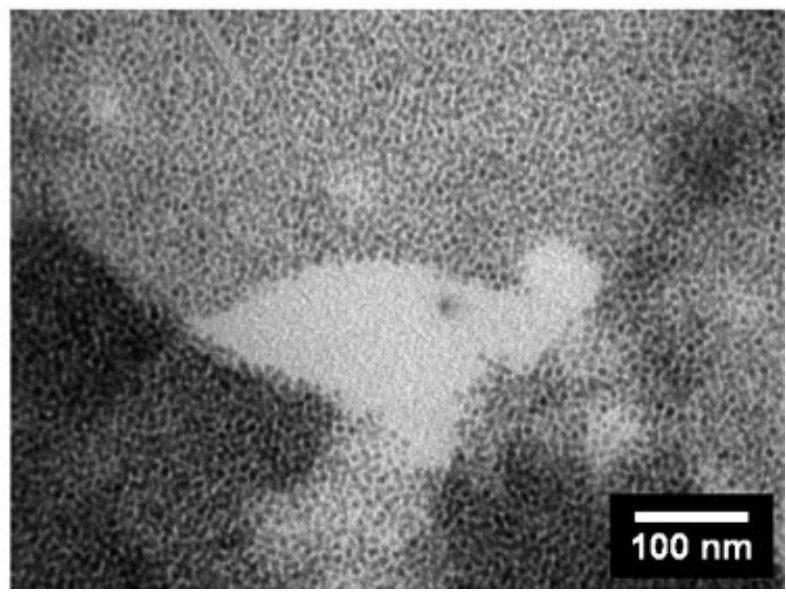

Figure 2. TEM images of nanocrystals deposited by drop-casting on TEM grids after a) slow and b) fast evaporation of the solvent. 
have been brought into conformal contact with the nanocrystal film for $30 \mathrm{~s}$ and then peeled away. The quality of the QD layers formed on the PDMS surface is reflective of the morphology of the QD film on the glass substrate. In other words, the quality of the QD layers depends on the concentration of the nanocrystal solution and the nature of their assembly on the glass surface. The inked PDMS stamp has then been brought into conformal contact with a small-molecule organic layer for $1 \mathrm{~min}$. Subsequently, the stamp has been peeled away from the layer. This leads to the transfer of the nanoparticle arrays onto the organic substrate. Figure 3 compares confocal microscopy images of QDs transferred onto a CBP layer by $\mu \mathrm{CP}$ doubletransfer from glass substrates prepared by slow (Fig. 3a) and fast evaporation (Fig. 3b) of the solvent. The morphologies of the $50 \mu \mathrm{m} \times 150 \mu \mathrm{m}$ QD stripes prepared by the two methods are strikingly different; relatively uniform stripes have been obtained for the sample fabricated by slow evaporation of the solvent (Fig. 3a), whereas very inhomogeneous morphologies have been observed for samples fabricated by fast evaporation of the solvent (Fig. 3b). This further corroborates the efficacy
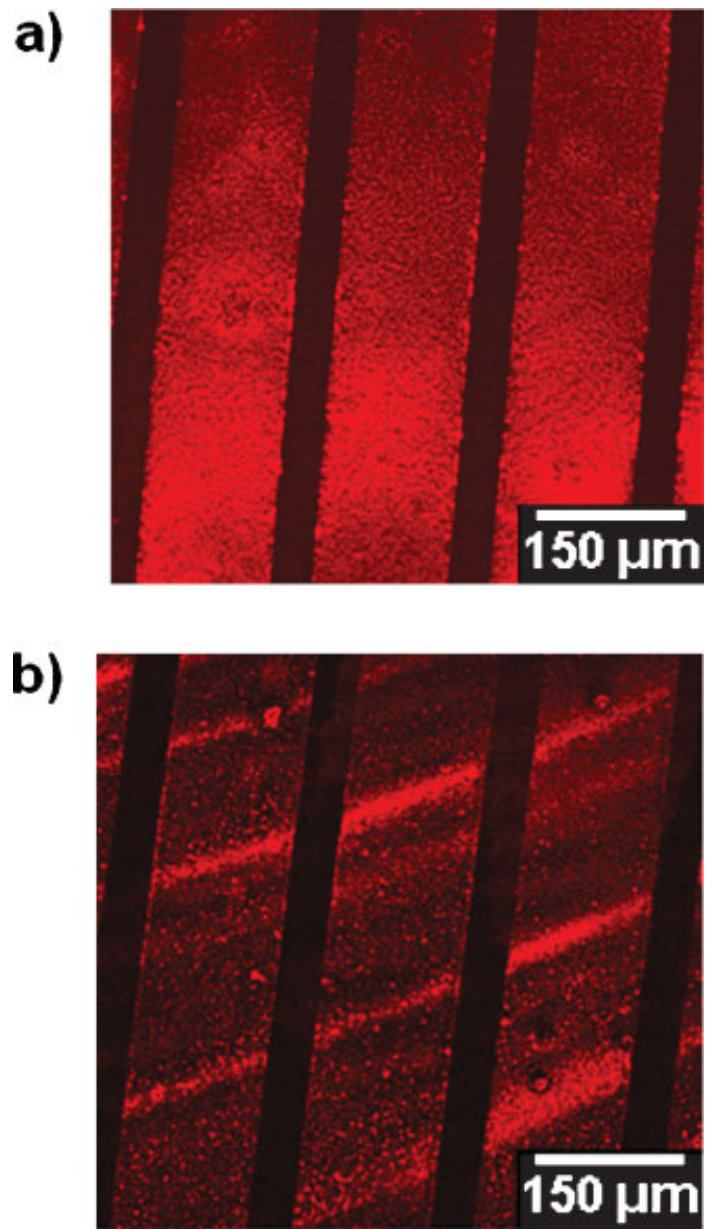

Figure 3. Confocal microscopy images of QDs transferred by doubletransfer $\mu \mathrm{CP}$ onto CBP organic substrates. a) QDs on CBP transferred from a glass substrate after slow evaporation of the solvent; b) QDs on $C B P$ transferred from a glass substrate after fast evaporation of the solvent. of slow solvent evaporation in achieving the fabrication of highly homogeneous films of colloidal QDs on the micrometer scale.

Figure 4 shows atomic force microscopy (AFM) and scanning electron microscopy (SEM) images of QD layers transferred onto CBP from QD solutions of different concentrations. Upon increasing the concentration of QDs in toluene solution by 15 -fold, the thickness of the transferred layer can varied from only a few layers (Fig. 4a) to more than $100 \mathrm{~nm}$ (Fig. 4b). Figure 4e and $\mathrm{f}$ shows the corresponding confocal microscopy images of these samples. It is clearly apparent that changes in the thickness are reflected in the varying luminance intensities of the two samples.

The transfer mechanism involved in the deposition of QDs from glass substrates onto the stamp can be explained by considering the strong interactions between the surface of the PDMS stamp and the hydrophobic surfactants present on the colloidal nanocrystal surfaces. The origin of the interactions can likely be traced to the existence of highly mobile hydrophobic oligomers on the PDMS stamp surface. ${ }^{[18]}$ Moreover, PDMS has a very low surface energy of $21.6 \mathrm{dyn} \mathrm{cm}^{-1}$ $\left(1 \mathrm{dyn} \mathrm{cm}^{-1}=1 \times 10^{3} \mathrm{~N} \mathrm{~m}^{-1}\right),{ }^{[16]}$ which allows the transfer of QDs to higher surface energy substrates such as CBP layers. In this context, the organic material acts as glue for the nanocrystals. ${ }^{[19]}$ This method has been exploited to integrate QDs into a multilayer organic LED. In order to realize a hybrid LED that emits light only from the nanocrystals, a highsurface-coverage and compact QD layer needs to be deposited on top of the organic material. ${ }^{[5]}$ Consequently, a flat PDMS pad has been used to transfer QDs during the device fabrication process (Fig. 5).

The device structure (Fig. 6a, inset) consists of a thermally evaporated $N, N^{\prime}$-bis(naphthalen-1-yl)- $N, N^{\prime}$-bis(phenyl)benzidine $(\alpha$-NPD) HIL doped with 2,3,5,6-tetrafluoro-7,7,8,8tetracyano-quinodimethane (F4-TCNQ) and a CBP HTL. An $80 \mathrm{~nm}$ thick QD active layer has been transferred onto the organic substrate by the double-transfer technique reported above. After the deposition of the QD layer, a 2,9-dimethyl4,7-diphenyl-1,10-phenanthroline (BCP) hole-blocking layer (HBL), a tris(8-(hydroxyl-quinoline) aluminum $\left(\mathrm{Alq}_{3}\right)$ electron-transport layer (ETL), and metal electrodes have been evaporated to complete the fabrication of the device. In the proposed device, holes and electrons are delivered to the nanocrystal layer through the injection and transport layers.

Figure $6 \mathrm{~b}$ shows the electroluminescence (EL) spectrum and a picture of the working device, indicating that the emission in this device is dominated by band-edge emission from the QD layer. The PL spectrum of the nanocrystals is shown by a dashed line in the same figure. A small red-shift (less than $10 \mathrm{~nm}$ ) and broadening of the EL spectrum as compared to the PL data can be attributed to environmental effects such as energy and charge transfer among nanocrystals or between nanocrystals and organic molecules. The QD charging process can change with the film thickness or surface roughness owing to variations in the local space charges or because of local Joule heating. ${ }^{[20,21]}$ 
a)
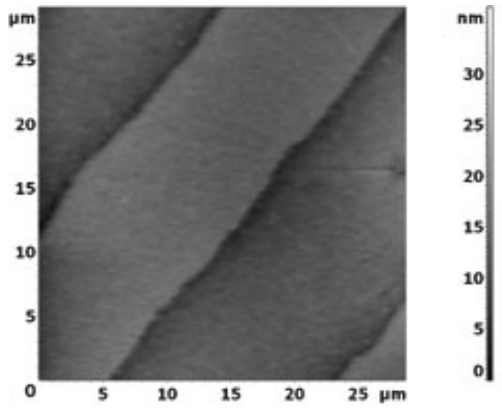

c)

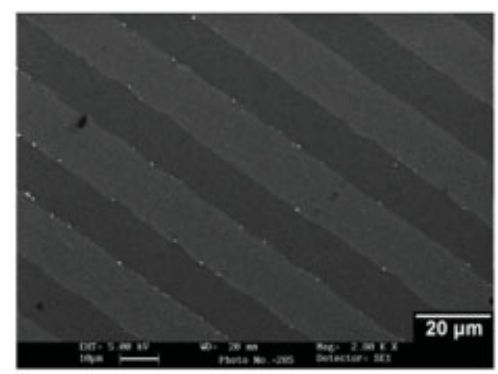

e)

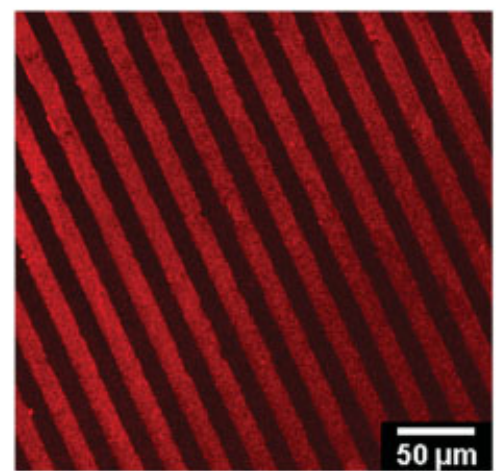

b)
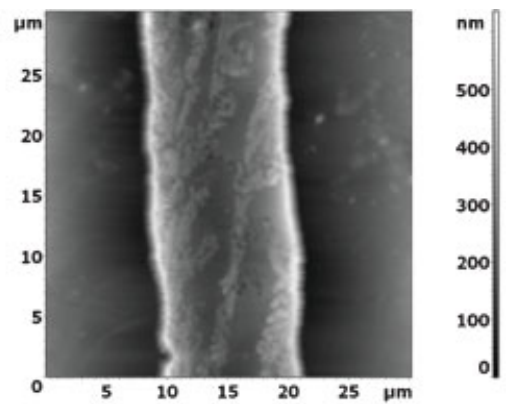

d)

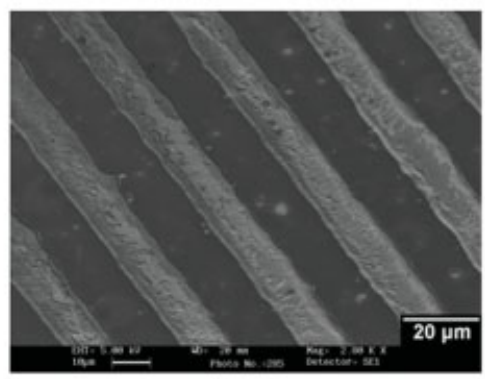

f)

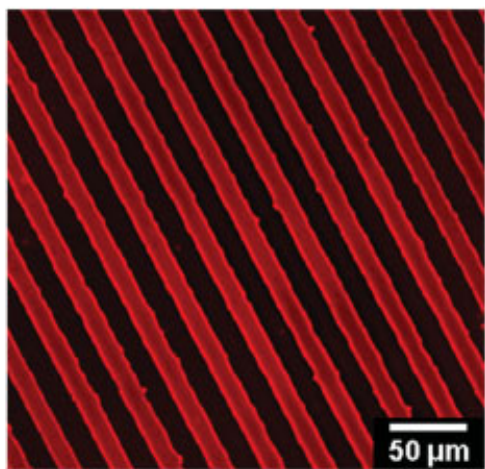

Figure 4. Atomic force microscopy (AFM), scanning electron microscopy (SEM), and confocal microscopy characterization of $12 \times 18 \mu \mathrm{m}^{2}$ stripes of QDs on CBP. a,b) AFM images of QD samples with a) a low concentration of QDs $\left(0.4 \mathrm{mg} \mathrm{mL}^{-1}\right)$ and b) a high concentration of QDs $\left(6 \mathrm{mg} \mathrm{mL}^{-1}\right)$. c,d) SEM images corresponding to (a) and (b), respectively; e,f) confocal microscopy images corresponding to (a) and (b), respectively.

The presence of a negligible $\alpha$-NPD $(420 \mathrm{~nm})$ emission suggests hole accumulation at the HIL/HTL and $\alpha-\mathrm{NPD} / \mathrm{CBP}$ interfaces owing to the alignment of the highest occupied molecular orbital (HOMO) levels. ${ }^{[22]}$ The HBL is effective in precluding the transfer of holes and excitons into the ETL,
$\mathrm{Alq}_{3}$, and thus inhibits emission from the ETL. ${ }^{[1]}$ As shown in the EL spectrum, emission from $\mathrm{Alq}_{3}(520 \mathrm{~nm})$ is negligible in this device. Figure 6a shows current-density versus voltage and luminescence versus voltage plots measured for these devices. The maximum brightness of the device is $90 \mathrm{cdm}^{-2}$ at a)

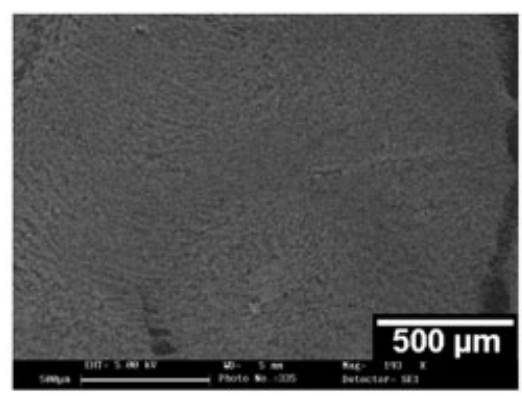

b)

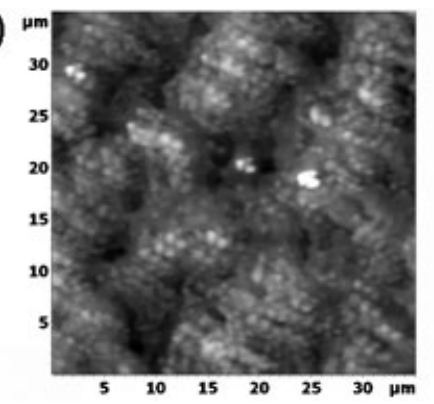

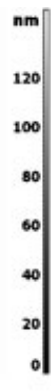

Figure 5. SEM and AFM characterization of a QD layer transferred from a flat PDMS pad onto CBP. a) SEM image of a QD layer showing a $2.5 \mathrm{~mm} \times 2.5 \mathrm{~mm}$ area. b) AFM morphology of the same sample. 
a)

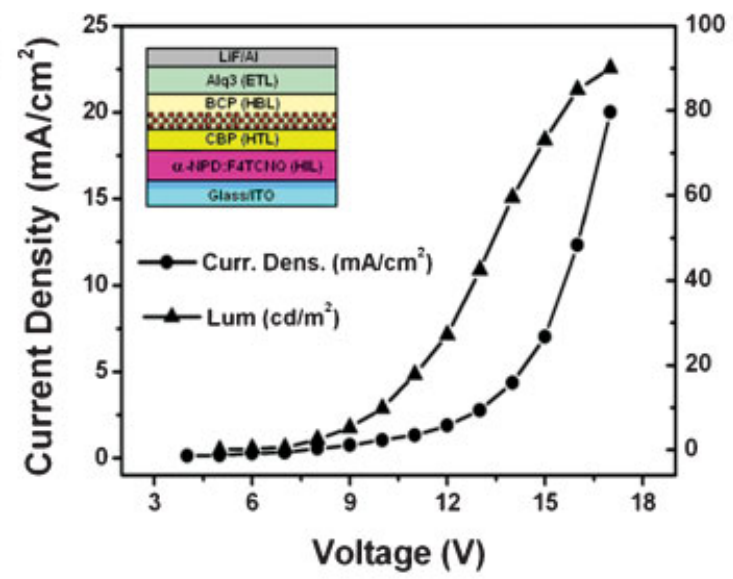

b)

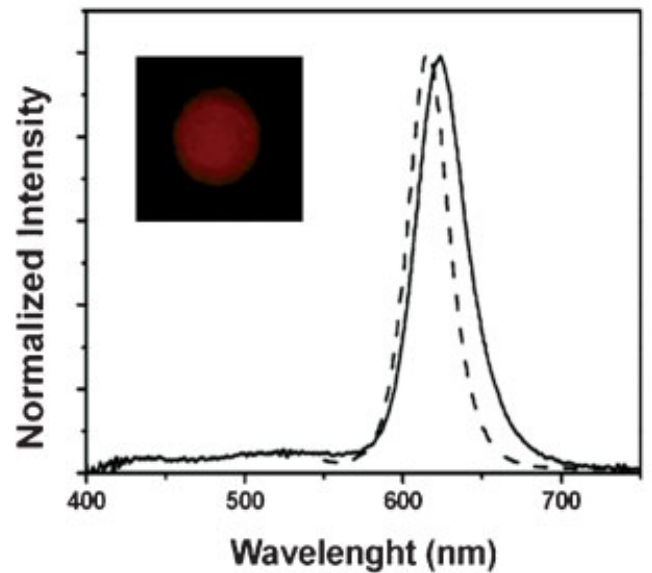

Figure 6. Structure and performance of a hybrid LED fabricated by $\mu \mathrm{CP}$ double transfer. a) Current-density-voltage and luminescence-voltage characteristics of the hybrid LED; the inset shows a schematic illustration of the device structure. b) Electroluminescence (solid line) and PL (dashed line) spectra; the inset shows a picture of a functional $1.5 \mathrm{~mm} \times 1.5 \mathrm{~mm}$ area device.

$20 \mathrm{~mA} \mathrm{~cm}^{-2}$, which corresponds to an external quantum efficiency of $0.15 \%$.

In conclusion, we have developed a totally dry technique for the deposition of colloidal semiconductor nanocrystals on organic substrates. This approach is fully compatible with current organic LED technology. It appears that the slow evaporation of drop-cast QD films is critical for the success of the transfer process. This novel approach has been utilized to fabricate a hybrid organic-inorganic red LED. The technique developed here enables us to separate the deposition of organic materials from the QD deposition process, thus enabling the fabrication of complex multilayered device structures without any restrictions on the choice of the organic material. Moreover, it is likely that this technique can be further optimized to deposit QDs with different emission wavelengths, such as blue, green, and red, on the same organic substrate, as well as for patterning QDs in pixels for future applications in flat-panel display technologies and micropatterned colloidal- nanocrystal-based microcavities. ${ }^{[23]}$ The flexibility and ease of this technique represents a significant advancement in the fabrication of novel QD-based LED structures.

\section{Experimental}

The PDMS mold was fabricated by casting PDMS onto a Si master. PDMS stamps were created by curing Sylgard 184 (a two-ingredient elastomer kit purchased from Dow Corning) on top of a Si master prepared by photolithography at $60^{\circ} \mathrm{C}$ for $5 \mathrm{~h}$. Colloidal semiconductor $\mathrm{CdSe} / \mathrm{ZnS}$ QDs that were maple red-orange in color with a crystal diameter of $5.11 \mathrm{~nm}$ were purchased from Evident Technologies.

Double Transfer: A $30 \mathrm{~nm}$ layer of CBP was thermally evaporated onto an indium tin oxide (ITO) substrate at a pressure of $2.0 \times 10^{-6} \mathrm{mbar}(1 \mathrm{mbar}=100 \mathrm{~Pa})$. A $50 \mu \mathrm{L}$ drop of $\mathrm{CdSe} / \mathrm{ZnS}$ QDs from Evident Technologies was deposited by drop casting on the surfaces of cleaned float glass substrates; different concentrations of QDs in toluene solution were used to prepare these drop-cast films. After the deposition process, the samples were placed in a desiccator and allowed to dry. PDMS stamps with different patterned line features were then brought into conformal contact with the QD films on the glass substrates for $30 \mathrm{~s}$. After careful removal from the surface, the inked PDMS pad was brought into conformal contact with the CBP substrate for $1 \mathrm{~min}$ and was then carefully peeled away.

The morphology of the patterned QD structures was characterized by confocal laser scanning microscopy (FluoView1000, Olympus) using an excitation wavelength of $405 \mathrm{~nm}$. AFM (Solver Pro, NT-MDT) in semi-contact mode and SEM (Stereoscan 440) were also used to characterize these structures.

Hybrid Electroluminescent Device: To fabricate the electroluminescent device, a $50 \mathrm{~nm}$ thick HIL, a $30 \mathrm{~nm}$ thick HTL (comprising $\alpha$-NPD doped with F4-TCNQ), and CBP were sequentially evaporated onto cleaned and oxygen-plasma-treated ITO substrates. Subsequently, a QD layer was deposited onto the organic materials by the double-transfer technique using a flat PDMS pad. Finally, a $15 \mathrm{~nm}$ HBL composed of BCP and a $20 \mathrm{~nm}$ ETL comprising $\mathrm{Alq}_{3}$ were deposited by thermal evaporation. All the organic materials were thermally evaporated at a rate of $1 \mathrm{As}^{-1}$ at a pressure of $2 \times 10^{-6} \mathrm{mbar}$. $0.5 \mathrm{~nm} \mathrm{LiF}$ and $200 \mathrm{~nm} \mathrm{Al}$ electrodes were evaporated through a contact shadow mask at a pressure of $4 \times 10^{-6}$ mbar.

The EL spectra and luminescence were measured using an OL 770 spectroradiometer (Optronic Laboratories). Current-voltage characteristics was measured using a Keithley 2400 source measurement unit. All the measurements were performed at room temperature in air without any protection from the ambient atmosphere.

Received: June 20, 2007 Revised: October 11, 2007

[1] S. Coe, W. K. Woo, M. G. Bawendi, V. Bulović, Nature 2002, 420, 800.

[2] Y. Q. Li, A. Rizzo, M. Mazzeo, L. Carbone, L. Manna, R. Cingolani, G. Gigli, J. Appl. Phys. 2005, 97, 113501.

[3] Y. Q. Li, A. Rizzo, R. Cingolani, G. Gigli, Adv. Mater. 2006, 18, 2545.

[4] S. Chaudhary, M. Ozkan, W. C. W. Chan, Appl. Phys. Lett. 2004, 84, 2925.

[5] S. Coe-Sullivan, W. K. Woo, J. S. Steckel, M. G. Bawendi, V. Bulović, Org. Electron. 2003, 4, 123

[6] A. Rizzo, Y. Q. Li, S. Kudera, F. Della Sala, M. Zanella, W. J. Parak, R. Cingolani, L. Manna, G. Gigli, Appl. Phys. Lett. 2007, 90, 051106. 


\section{ADVANCED}

[7] J. S. Steckel, P. Snee, S. Coe-Sullivan, J. P. Zimmer, J. E. Halpert, P. Anikeeva, L. A. Kim, V. Bulović, M. G. Bawendi, Angew. Chem. Int. Ed. 2006, 45, 5796 .

[8] B. O. Dabbousi, J. Rodriguez-Viejo, F. V. Mikulec, J. R. Heine, H. Mattoussi, R. Ober, K. F. Jensen, M. G. Bawendi, J. Phys. Chem. B 1997, 101, 9463.

[9] P. Reiss, J. Bleuse, A. Pron, Nano Lett. 2002, 2, 781.

[10] S. Kudera, M. Zanella, C. Giannini, A. Rizzo, Y. Q. Li, G. Gigli, R. Cingolani, G. Ciccarella, W. Spahl, W. J. Parak, L. Manna, Adv. Mater. 2007, 19, 548

[11] X. M. Lin, H. M. Jaeger, C. M. Sorensen, K. J. Klabunde, J. Phys. Chem. B 2001, 105, 3353.

[12] M. A. Islam, I. P. Herman, Appl. Phys. Lett. 2002, 80, 3823.

[13] V. Santhanam, J. Liu, R. Agarwal, R. P. Andres, Langmuir 2003, 19, 7881.

[14] V. Santhanam, R. P. Andres, Nano Lett. 2004, 4, 41.
[15] S.-S. Bae, D. K. Lim, J.-I. Park, W.-R. Lee, J. Cheon, S. Kim, J. Phys. Chem. B 2004, 108, 2575 .

[16] Y. Xia, G. M. Whitesides, Annu. Rev. Mater. Sci. 1998, 28, 153.

[17] S. Coe-Sullivan, Ph. D. Thesis, Massachusetts Institute of Technology 2005.

[18] H. Schmid, H. Wolf, R. Allenspach, H. Riel, S. Karg, B. Michel, E. Delamarche, Adv. Funct. Mater. 2003, 13, 145.

[19] X. Yan, J. Yao, G. Lu, X. Chen, K. Zhang, B. Yang, J. Am. Chem. Soc. 2004, 126, 10510.

[20] J. L. Zhao, J. Y. Zhang, C. Y. Jiang, J. Bohhenberger, T. Basche, A Mews, J. Appl. Phys. 2004, 96, 3206.

[21] B. O. Dabbousi, M. G. Bawendi, O. Onitsuka, M. F. Rubner, Appl. Phys. Lett. 1995, 66, 1316.

[22] C. Adachi, R. Kwong, S. R. Forrest, Org. Electron. 2001, 2, 37.

[23] L. Martiradonna, L. Carbone, M. De Giorgi, L. Manna, G. Gigli, R. Cingolani, M. De Vittorio, Appl. Phys. Lett. 2006, 88, 181108. 